\title{
Lead photocathodes
}

\author{
J. Smedley and T. Rao \\ Brookhaven National Laboratory, Upton, New York 11973, USA
}

J. Sekutowicz

DESY, Notkestrasse 85, 22603 Hamburg, Germany

(Received 18 September 2007; published 30 January 2008)

\begin{abstract}
We present the results of our investigation of lead as a suitable photocathode material for superconducting rf injectors. Quantum efficiencies $(\mathrm{QE})$ have been measured for a range of incident photon energies and compared to predictions from the three-step model of photoemission. A variety of cathode preparation methods have been used, including various lead plating techniques on a niobium substrate. The effects of operating at ambient and cryogenic temperatures and different vacuum levels on the cathode QE have also been studied.
\end{abstract}

DOI: 10.1103/PhysRevSTAB.11.013502

PACS numbers: 29.25.Bx, 79.60.-i

\section{INTRODUCTION}

Several projects now being considered require injectors capable of delivering significant average current $(>1 \mathrm{~mA})$ with near-continuous operation [1-4]. Some of these applications also require significant peak current and would benefit from high ( $>20 \mathrm{MV} / \mathrm{m}$ ) accelerating gradients. For these applications, a photoinjector based on superconducting technology is a leading contender.

Designing a superconducting photoinjector entails many challenges. As with any photoinjector, the choice of cathode material is of critical importance. The cryogenic environment of the superconducting cavity places additional considerations on the cathode choice compared to roomtemperature copper cavities. Any cathode that is not itself a superconductor may need to be thermally isolated from the cavity, and must be mounted in such a way as to minimize rf loss in the cavity. A solution to these isolation problems has been proposed by Michalke et al. [5] and investigated by Jensen and co-workers at Rossendorf [6], using an rf choke-joint on the back wall of the cavity. This is an attractive solution, as it potentially enables the use of nearly the entire range of cathodes developed for roomtemperature cavities. Chemical contamination of the superconducting cavity may also be a problem for some cathode materials. To address this concern, a group at Brookhaven National Laboratory [7] is investigating the use of a diamond capsule to enclose the cathode, with the diamond acting both as an electron multiplier (via secondary emission) and as a barrier to prevent contamination of the cavity by the cathode and contamination of the cathode by the cavity.

Another option is to use a superconductor as the cathode. This eliminates the need for isolation and simplifies the overall design. These cathodes can be expected to exhibit the benefits and drawbacks of traditional metal cathodes nearly unlimited operating life and low quantum efficiency $\left(<10^{-3}\right)$. The niobium that comprises the cavity walls is the obvious first choice, and a cavity utilizing a niobium cathode has been built and tested at BNL [8]. The low quantum efficiency (QE) measured for $\mathrm{Nb}$ [9] does not lend itself to producing $>1 \mathrm{~mA}$ current with existing laser systems. Lead has been suggested as a possible alternative photocathode $[1,10,11]$. Lead is a type I superconductor commonly used in cavities for ion accelerators, with a critical temperature of $7.2 \mathrm{~K}$ and a critical magnetic field of $80 \mathrm{mT}$. This low critical field limits the accelerating gradient achievable in a cavity constructed entirely of lead. For this reason, we intend to coat only the cathode region of a niobium cavity with lead. As the magnetic field on the beam axis is typically small, we do not expect the maximum electric field in the cavity to be impacted by the lead coating. This has been verified in recent tests at Jefferson Laboratory [12].

The first section of this paper details the theoretical model of photoemission from lead, based on Spicer's three-step model of photoemission [13]. The second section addresses the experimental measurement of the quantum efficiency of various lead coatings, both at room and cryogenic temperatures. Portions of the experimental section were previously reported [14] in conference proceedings, and are included here for completeness.

\section{PHOTOEMISSION MODEL}

Spicer's three-step model of photoemission [13] has been used to predict the quantum efficiency and the energy spectrum of emitted electrons for a lead photocathode as a function of the photoenergy of the incident light. This model treats photoemission as a sequence of three independent processes: photon absorption/electron excitation, electron migration to the surface, and electron escape from the surface. Each process has an associated probability. The first step calculates the probability of absorption of a photon and excitation of an electron to an energy $E$. The second step calculates the probability that an electron of energy $E$ will reach the surface without scattering. For the purposes of this model, electron-electron scattering is con- 
sidered to be a loss mechanism, as neither the scattering electron nor the scattered electron are likely to retain energy sufficient to escape the material. The third step calculates the probability that the electron of energy $E$ will have sufficient momentum perpendicular to the surface to escape. The product of these probabilities represents the probability that an electron of energy $E-\Phi$ will be emitted from the surface. This information is useful, as it provides the energy spectrum of the emitted electrons (often called the energy distribution curve or EDC), which in turn is linked to the "thermal" emittance of the photocathode [15]. The QE is then the area under the EDC. In the following analysis, we have tried to keep our notation consistent with other recent work on this topic $[16,17]$.

\section{A. Step 1: Photon absorption and electron excitation}

Obtaining the probability that an incident photon of energy $h \nu$ will produce an electron of energy $E$ inside the material is the goal of the first step. This is actually the product of two probabilities - the probability that the photon is absorbed (not reflected), and the probability that the photon will be absorbed by an electron of energy $E-h \nu$. Following Spicer, we assume that the probability of an electron of energy $E-h \nu$ absorbing the photon is based solely on the product of the number of available electrons at energy $E-h \nu$ and the number of available empty states at energy $E$. The number of electrons at energy $E-h \nu$ is $N(E-h \nu) F(E-h \nu, T)$, where $N(E)$ is the density of electron states (DOS), and $F(E, T)$ is the Fermi-Dirac distribution:

$$
F(E, T)=\frac{1}{1+e^{\left(E-E_{F}\right) / k_{B} T}} .
$$

Similarly, the number of empty states at energy $E$ is $N(E)(1-F(E, T)$. The probability for step one is then

$$
P(E, h \nu, T)=[1-R(\nu)] \frac{N(E)[1-F(E, T)] N(E-h \nu) F(E-h \nu, T)}{\int_{0}^{\infty} d E^{\prime} N\left(E^{\prime}\right)\left[1-F\left(E^{\prime}, T\right)\right] N\left(E^{\prime}-h \nu\right) F\left(E^{\prime}-h \nu, T\right)}
$$

Here $R(\nu)$ is the reflectivity, and the denominator represents a normalization based on the assumption that all photons which are not reflected are absorbed by electrons in the metal. For the remainder of this work, we will use the Fermi-Dirac function at zero temperature, thus all states below the Fermi energy are filled, and all states above are empty. This yields the simplification:

$$
P(E, h \nu)=[1-R(\nu)] \frac{N(E) N(E-h \nu)}{\int_{E_{f}}^{E_{f}+h \nu} d E^{\prime} N\left(E^{\prime}\right) N\left(E^{\prime}-h \nu\right)} .
$$

In order to calculate this probability for lead, the electron density of states was obtained from the Naval Research

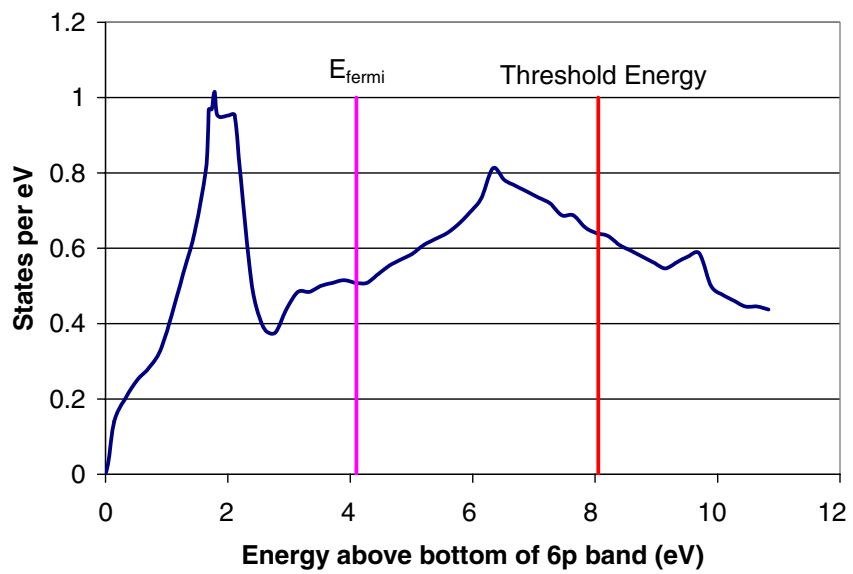

FIG. 1. (Color) Density of electron states of lead [18]. Note that zero energy is taken to be the bottom of the $6 p$ band.
Laboratory structures database [18]. The portion of the DOS relevant for this work is shown in Fig. 1, with the location of the Fermi energy and the emission threshold $\left(E_{T}=E_{f}+\Phi\right)$ relative to the bottom of the lead $6 p$ band. The optical constants used to calculate the reflectivity for lead and the "theoretical" work function $\left(\Phi_{0}=3.95 \mathrm{eV}\right)$ were obtained from the literature $[19,20]$.

\section{B. Step 2: Transit to surface}

The probability that an electron reaches the surface of the material depends on the depth at which it was excited and its mean-free path with respect to electron-electron scattering. Note that this treatment ignores electronphonon scattering, as such scattering is generally nearly elastic. Spicer [13] gives the fraction of the electrons which reach the surface without scattering as

$$
F_{e-e}(E, h \nu)=\frac{1}{1+\frac{\lambda_{\mathrm{opt}}}{\lambda_{e^{-e}}}} C\left(\frac{\lambda_{e^{-e}}}{\lambda_{\mathrm{opt}}}, D(E)\right)
$$

$\lambda_{\text {opt }}$ is the photon mean-free path, $\lambda_{\text {opt }}=\lambda /(4 \pi k)$, where $k$ is the imaginary part of the index of refraction. $\lambda_{e-e}$ is the electron mean-free path for electron-electron scattering. Here $C$ is a correction factor which accounts for the fact that the electron trajectory is not necessarily normal to the material surface. We will see in step three that the trajectory must be near normal for an electron to be able to escape, so this correction is expected to be small. This factor is 


$$
\begin{aligned}
& C\left(\frac{\lambda_{e-e}}{\lambda_{\mathrm{opt}}}, D(E)\right)= \frac{\frac{\lambda_{e^{-e}}}{\lambda_{\mathrm{opt}}}+1}{\frac{\lambda_{e^{-e}}}{\lambda_{\mathrm{opt}}}}-\frac{\frac{\lambda_{e^{-} e}}{\lambda_{\mathrm{opt}}}+1}{2 D\left(\frac{\lambda_{e-e}}{\lambda_{\mathrm{opt}}}\right)^{2}} \\
& \times \ln _{\frac{\lambda_{e^{-} e}}{\lambda_{\mathrm{opt}}}}+1-2 \frac{\lambda_{e^{-e}}}{\lambda_{\mathrm{opt}}}+1 \\
& \lambda_{e_{\mathrm{opt}}} D
\end{aligned} .
$$

$D(E)$ is the semiclassical threshold function, described by Eq. (9).

To calculate $\lambda_{e-e}$ using Spicer's method, we require a measured value for the mean-free path at a single energy. This data was not available for lead in the energy range of interest here. We therefore follow the method of Jensen [17] and Lugovskoy [21]. This method calculates the "hot electron" lifetime from first principles, assuming a freeelectron DOS. The expression for the lifetime is

$$
\begin{aligned}
\tau_{e-e}(E) & =\frac{8 \hbar K_{s}^{2}}{\alpha_{\mathrm{fs}}^{2} \pi m_{e} c^{2}}\left(\frac{E}{k_{B} T}\right)^{2}\left[\left(1+\left(\frac{E-E_{f}}{\pi k_{B} T}\right)^{2}\right) \gamma\left(\frac{2 k}{q_{0}}\right)\right]^{-1} \\
\gamma(x) & =\frac{x^{3}}{4}\left(\tan ^{-1} x+\frac{x}{1+x^{2}}-\frac{\tan ^{-1}\left(x \sqrt{2+x^{2}}\right)}{\sqrt{2+x^{2}}}\right) \\
q_{0} & =\sqrt{3 n e^{2} / 2 \varepsilon_{0} E_{f} K_{s}} \quad k=m_{e} v / \hbar=\sqrt{2 E m_{e}} / \hbar .
\end{aligned}
$$

Here energy is measured from the bottom of the freeelectron DOS, so $E_{f}=9.27 \mathrm{eV} . m_{e}$ is the electron mass, $\alpha_{\mathrm{fs}}$ is the fine-structure constant. $K_{s}$ is a correction factor that accounts for electron screening; the value for lead was found [17] to be 16.28. $n$ is the density of conduction electrons and is $1.3 \times 10^{23} / \mathrm{cc}$ for lead. Note that for $E-E_{f} \gg k_{B} T$ (our case), the expression for $\tau_{e-e}$ is essentially independent of temperature. The mean-free path can be obtained from the lifetime by multiplying by the velocity, $v=\left(2 E / m_{e}\right)^{1 / 2}$. A plot of $\tau_{e^{-e}}$ and $\lambda_{e^{-e}}$ as a function of hot electron energy above the Fermi level is shown in Fig. 2.

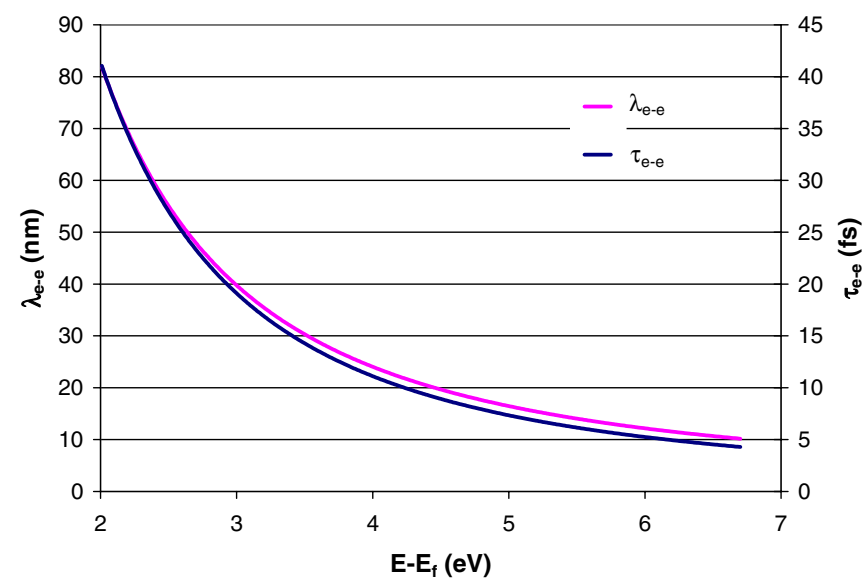

FIG. 2. (Color) Calculated electron mean-free path $\left(\lambda_{\mathrm{e}-\mathrm{e}}\right)$ and lifetime $\left(\tau_{\mathrm{e}-\mathrm{e}}\right)$.

\section{Step 3: Escape}

To escape the surface, the perpendicular component of the electron's momentum must be

$$
\frac{\hbar k_{\perp}^{2}}{2 m}>E_{T}
$$

Here the zero of energy is taken to be the bottom of the $6 p$ band in lead (shown in Fig. 1). From this reference, $E_{f}$ is $4.1 \mathrm{eV}$. The threshold energy may be modified by the applied field on the cathode due to the Schottky effect. In this case, $E_{T}=E_{f}+\Phi_{0}-\Phi_{\text {Schottky }}$, with $\Phi_{\text {Schottky }}=3.7947 \times 10^{-5} \sqrt{E(V / m)} \mathrm{eV}$. We define $\Phi_{\text {eff }}=\Phi_{0}-\Phi_{\text {Schottky }}$. Equation (7) defines a maximum angle the electron can have with respect to the surface normal:

$$
\theta_{\max }(E)=\cos ^{-1}\left(\frac{E_{T}}{E}\right)^{1 / 2}
$$

The excitation process is assumed to be isotropic, therefore the probability that an electron of energy $E$ has a trajectory which meets the criterion for escape is

$$
\begin{aligned}
D(E) & =\frac{1}{4 \pi} \int_{0}^{\theta_{\max }} \sin \theta^{\prime} d \theta^{\prime} \int_{0}^{2 \pi} d \varphi=\frac{1}{2}\left(1-\cos \theta_{\max }\right) \\
& =\frac{1}{2}\left(1-\left(\frac{E_{T}}{E}\right)^{1 / 2}\right) .
\end{aligned}
$$

\section{Energy distribution curve and quantum efficiency}

The EDC is just the product of the probabilities calculate in each step:

$$
N(E, h \nu)=P(E, h \nu) F_{e-e}(E, h \nu) D(E) .
$$

The EDC represents the number of electrons produced with energy $E$ per incident photon of energy $h \nu$, in units of electrons per photon per $\mathrm{eV}$. energy distribution curves for lead were calculated for a number of $h \nu$ values and some of the results are shown in Fig. 3. The QE at a particular photon energy is simply the area under the EDC plot for that photon energy:

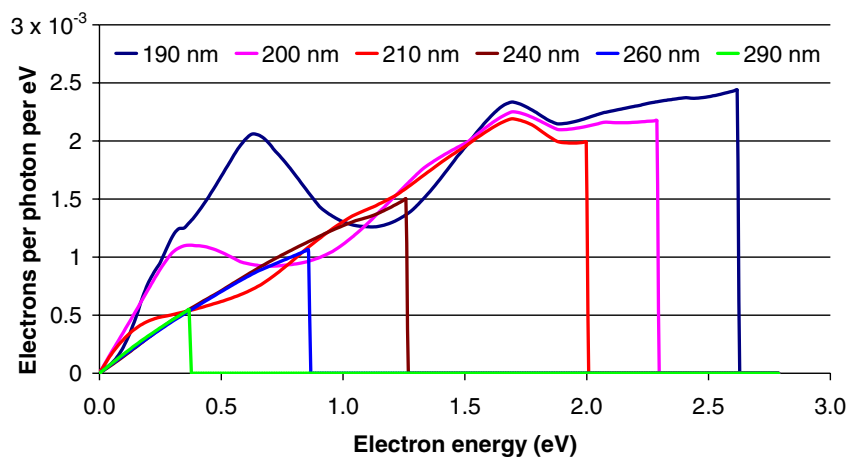

FIG. 3. (Color) Calculated energy distribution curves for lead irradiated with various wavelengths of light. 


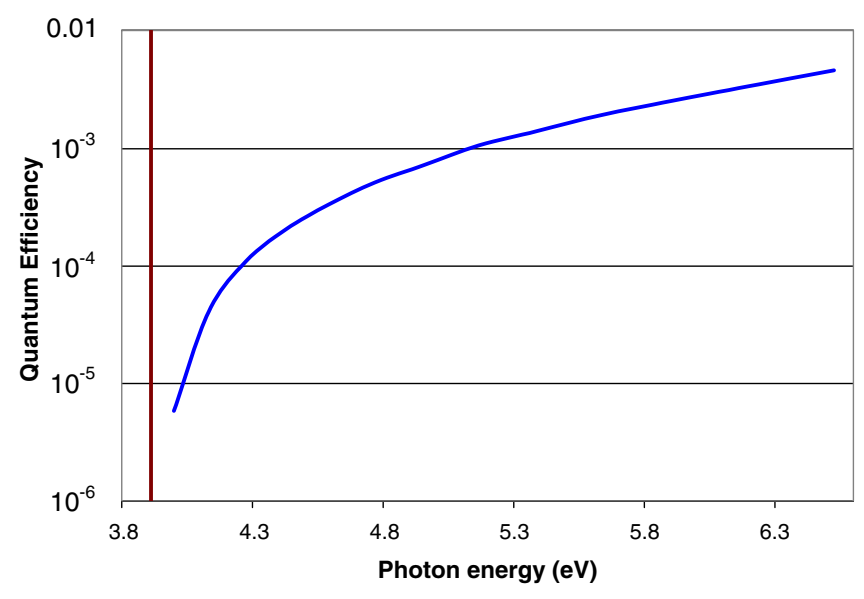

FIG. 4. (Color) Predicted QE for a lead photocathode as a function of incident photon energy. The vertical line represents the expected work function accounting for the Schottky effect $(3.91 \mathrm{eV})$.

$$
\mathrm{QE}(h \nu)=\int_{E_{f}+\Phi_{\mathrm{eff}}}^{E_{f}+h \nu} P(E, h \nu) F_{e-e}(E, h \nu) D(E) d E .
$$

Equation (11) was used to generate the theoretical dependence of QE on $h \nu$ and is shown in Fig. 4. For this case, the applied electric field was taken to be $1 \mathrm{MV} / \mathrm{m}$ resulting in a Schottky reduction of $0.04 \mathrm{eV}$ and leading to a value of $3.91 \mathrm{eV}$ for $\Phi_{\text {eff }}$.

\section{LEAD CATHODES}

\section{A. Preparation of cathodes}

Five distinct types of lead cathodes were investigated in this work - four methods of plating lead along with a solid, polished lead sample. In all cases the cathodes/substrates are $9 \mathrm{~mm}$ in diameter and $8 \mathrm{~mm}$ thick. The substrates were mechanically polished with Beuhler diamond polishing compounds. Nine, six, and one micron polishing compounds were used, resulting in a finish with scratches on the order of one micron wide. The substrates were ultrasonically cleaned in hexane for 20 minutes to remove any remnant of the polishing compound, and then dried with nitrogen.

Electroplated samples were prepared at Stony Brook University (SBU) on both copper and niobium substrates. The polished substrates were electroplated with lead by a procedure developed for use on superconducting cavities for heavy ion accelerators [22]. The plating solutions are based on methane-sulfonic acid chemistry and are created from commercially available products [23]. Each copper substrate was immersed in plating solution and flashed 4 times for several seconds with $10 \mathrm{~mA} / \mathrm{cm}^{2}$ plating current to prepare the surface. The current was then lowered to $2 \mathrm{~mA} / \mathrm{cm}^{2}$, corresponding to a plating rate of $10 \mu \mathrm{m} /$ hour, and the cathode was plated with $8 \mu \mathrm{m}$ of lead. The cathodes were then rinsed with deionized water and dried with a stream of nitrogen gas. The procedure for plating niobium is identical to that for copper, except that the coating thickness is $2 \mu \mathrm{m}$.

The bulk lead cathode was prepared from cylindrical stock obtained from Goodfellow (99.95\% purity). The surface was mechanically polished with Beuhler diamond polishing compounds, using the procedure for polishing substrates. The ultrasonic cleaning was insufficient to completely remove the diamond particulate from the soft lead surface. Scanning electron microscope (SEM) images taken after the photoemission measurements revealed diamond inclusions covering roughly $5 \%$ of the cathode surface, likely imbedded during polishing [see Figs. 6(d) and 7(d)].

The vacuum-deposited (evaporated) sample was prepared at SBU in a vacuum evaporator, with a background pressure of $5 \mu$ Torr and a deposition time of 9 minutes. The coating thickness was $6 \mu \mathrm{m}$. A polished copper cathode was used as a substrate. Subsequent evaporations have been prepared on niobium substrates, but have not yet been tested.

The magnetron sputtered sample was coated at the Andrzej Soltan Institute for Nuclear Studies, using a cylindrical cathode in the presence of 3 mTorr of argon. The niobium substrate was placed at a distance of $3 \mathrm{~cm}$ from the cathode and the current discharge was set at $100 \mathrm{~mA}$. Such conditions ensure stable discharge without any surface melting of the cathode. Total deposition time was $60 \mathrm{mi}-$ nutes and the estimated thickness of the layer was $4 \mu \mathrm{m}$.

The arc-deposited sample was also prepared at the Andrzej Soltan Institute for Nuclear Studies with a planar cathode under UHV conditions. This process has a high ionization ratio of metallic plasma, higher energy ions in comparison with the magnetron sputtering technique, and also a higher purity of the deposition process due to the absence of a working gas. A discharge current of $25 \mathrm{~A}$ provided stable arc operation. The deposition process was performed with an Aksenov-type magnetic filter connected to the plasma source in order to eliminate macrodroplets. The coating thickness was $1 \mu \mathrm{m}$ on a mechanically polished niobium substrate. Attempts to plate niobium substrates with machine-finished surfaces (not polished) via arc-deposition produced surfaces with less than complete lead coverage.

\section{B. Laser cleaning}

Laser cleaning with $248 \mathrm{~nm}$ light was used to improve the QE of all of the samples measured. The $248 \mathrm{~nm}$ light was provided by a $\mathrm{KrF}$ excimer (GAM Laser EX5), with a pulse duration of $5 \mathrm{~ns}$ and a repetition rate of $20 \mathrm{~Hz}$. The change in surface morphology induced by various energy densities of $248 \mathrm{~nm}$ light was determined using an electroplated lead sample (shown in Fig. 5). After irradiation, the cathode was removed from the vacuum and observed with an optical microscope and a SEM. The SEM was used to 

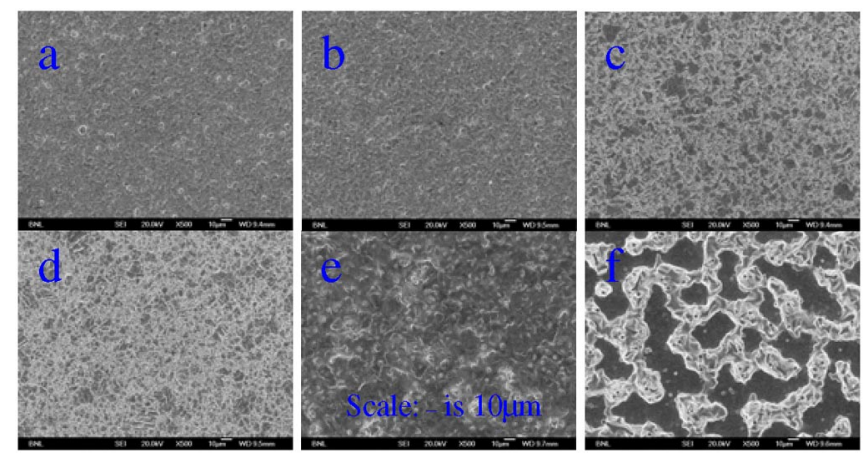

FIG. 5. (Color) Surface structure of a lead coated cathode after $248 \mathrm{~nm}$ laser irradiation: (a) no laser (b) 0.11 (c) 0.26 (d) 0.52 (e) 1.1 (f) $1.8 \mathrm{~mJ} / \mathrm{mm}^{2}$.

perform an analysis of the surface composition via x-ray fluorescence, as well as the surface structure. The first observable change in the surface morphology [Fig. 5(c)] occurred at an energy density of $0.26 \mathrm{~mJ} / \mathrm{mm}^{2}$. An energy density of $1.8 \mathrm{~mJ} / \mathrm{mm}^{2}$ was found to locally melt the coating and expose the substrate - the dark areas in Fig. 5(f) correspond to the copper substrate. Based on these results, an energy density of $0.2 \mathrm{~mJ} / \mathrm{mm}^{2}$ was chosen as the starting point for the cleaning.

The surfaces were each irradiated for $\sim 10$ minutes, with the laser operating at $20 \mathrm{~Hz}$. To avoid alignment difficulties, the region of the cathode exposed to the cleaning beam was significantly larger than the measurement area. For the solid lead, evaporated and electroplated samples, the cathode was cleaned a second time at an energy density of $0.4 \mathrm{~mJ} / \mathrm{mm}^{2}$. In both cases, little improvement was observed in the QE due to this increase. The samples were analyzed with an SEM after removal from the measure-
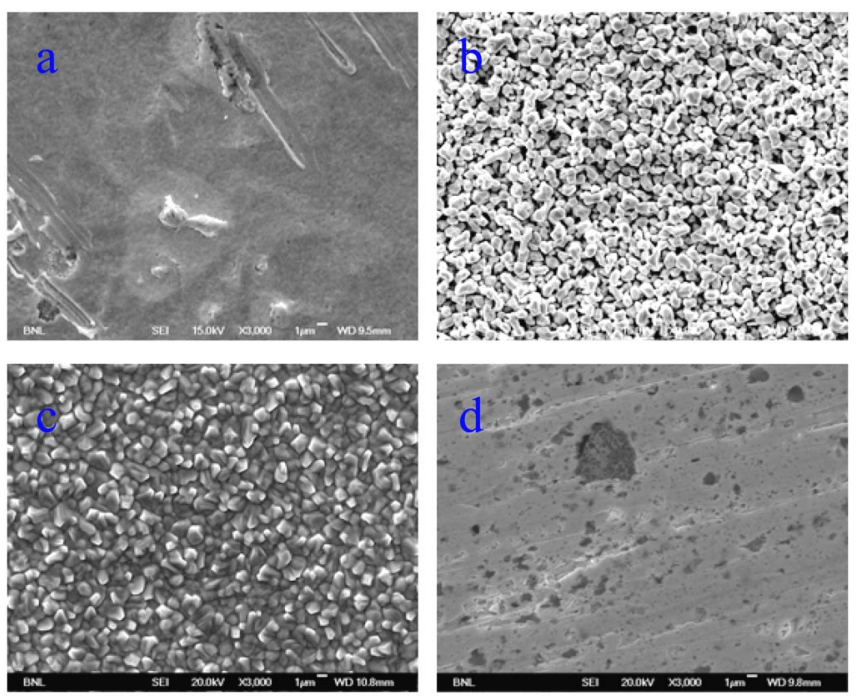

FIG. 6. (Color) Lead cathode surface morphology prior to laser cleaning: (a) arc-deposited, (b) sputtered, (c) evaporated, (d) polished solid lead.
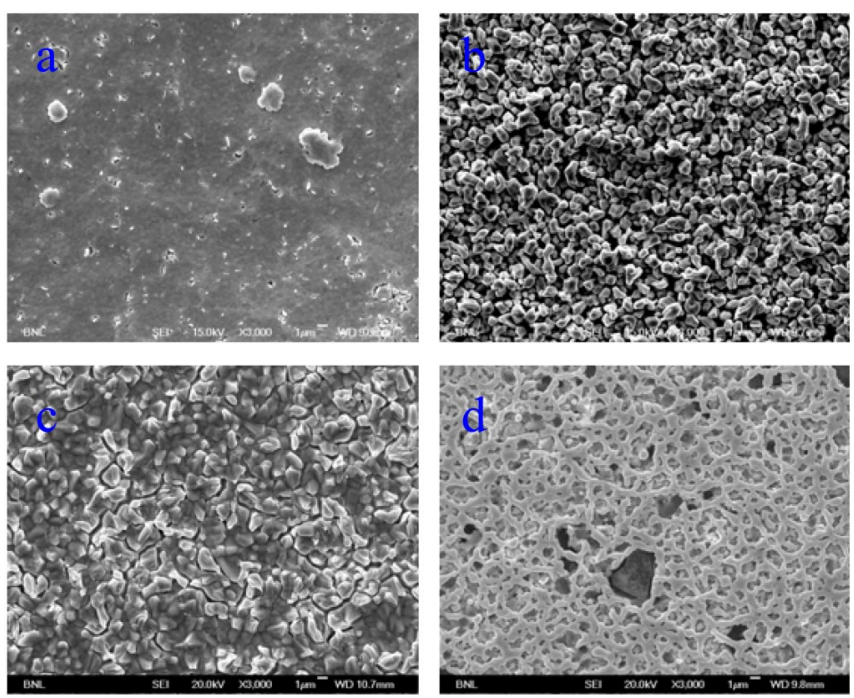

FIG. 7. (Color) Lead cathode surface morphology after irradiation with $0.2 \mathrm{~mJ} / \mathrm{mm}^{2}$ : (a) arc-deposited, (b) sputtered, (c) evaporated, (d) polished solid lead.

ment system. Figure 6 shows the surface structure for four of the cathodes before cleaning. Figure 7 shows the surface structure after cleaning. In all four cases, the x-ray fluorescence spectrum in the SEM showed only lead; this indicates that the coating was intact.

\section{PHOTOEMISSION MEASUREMENT}

A schematic of the photoemission measurement system is shown in Fig. 8. A deuterium light source (Ocean Optics DH-2000-S-DUV) is fiber coupled to a monochromator with a $300 \mu \mathrm{m}$ exit slit. The desired wavelength $\lambda$ is selected by the dial on the monochromator (Edmund DCM1-01). The output bandwidth is $2 \mathrm{~nm}$, measured with an Ocean Optics HR2000 spectrometer. A fused silica lens is used to focus the light on the cathode through a vacuum window and the anode mesh. The output of the monochromator is measured for each wavelength before and after each QE measurement, at a point after the lens but prior to the vacuum window, using a power meter (Newport 918-UV). For the QE measurement, the anode is held at a positive voltage, and the current is measured leaving the

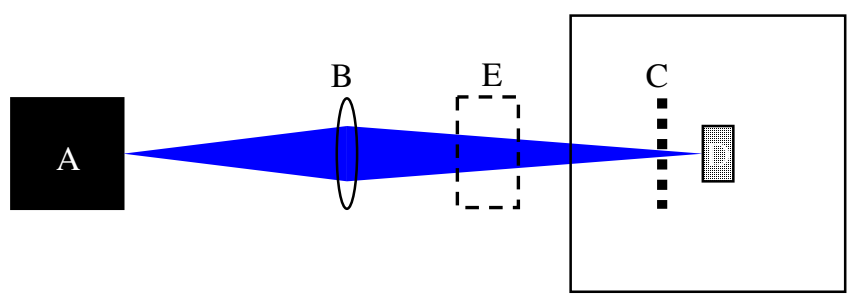

FIG. 8. (Color) Schematic of experimental arrangement, showing: (A) monochromator, (B) lens, (C) anode grid, (D) cathode, and $(\mathrm{E})$ location of power meter. 


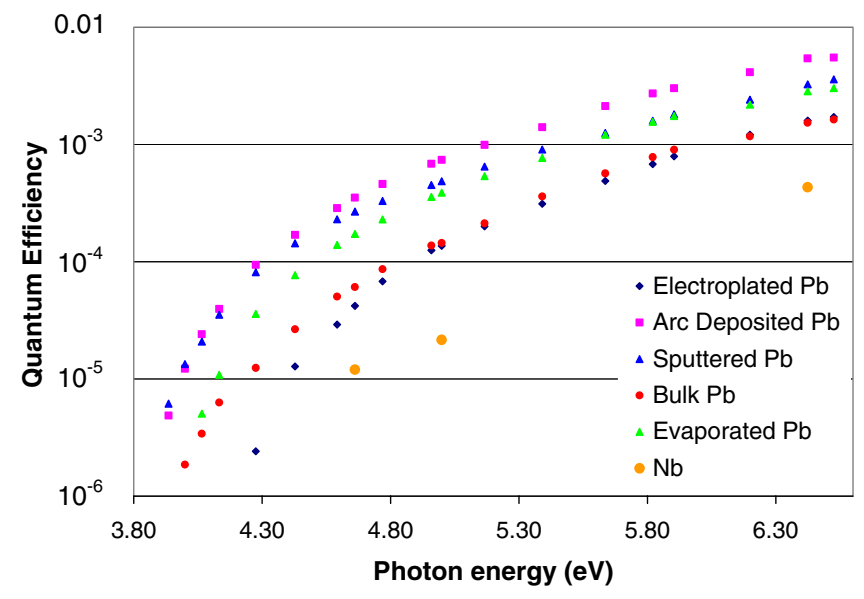

FIG. 9. (Color) Quantum efficiencies of various laser cleaned lead cathodes for photon energies between 3.9 and $6.5 \mathrm{eV}$.

cathode by a picoammeter (Keithley 487). The optical transmission of the vacuum window and the mesh are calibrated separately for each wavelength. Typical values for optical power $P$ (in a $2 \mathrm{~nm}$ band) are $10-100 \mathrm{nW}$, and typical values for current $I$ are 0.1 to $10 \mathrm{pA}$. For each wavelength, $\mathrm{QE}$ is calculated using the formula $\mathrm{QE}=$ $I(h \nu) /(P e)$, with $\nu=c / \lambda$.

The quantum efficiency of the five lead cathodes is shown in Fig. 9 as a function of incident photon energy. The niobium data is included for Ref. [9]. Figure 10 shows a comparison between the predicted and measured QE for the arc-deposited lead cathode. The theory line in the plot differs from that in Fig. 4 only in that the measured work function of $3.88 \mathrm{eV}$ was used. It should be noted that there are no fitting parameters in this model. In this light, the agreement between the best values measured for lead and the theory predictions is good. The difference in the work functions of the other lead cathodes cannot entirely account for the lower QE of these cathodes. Factors not

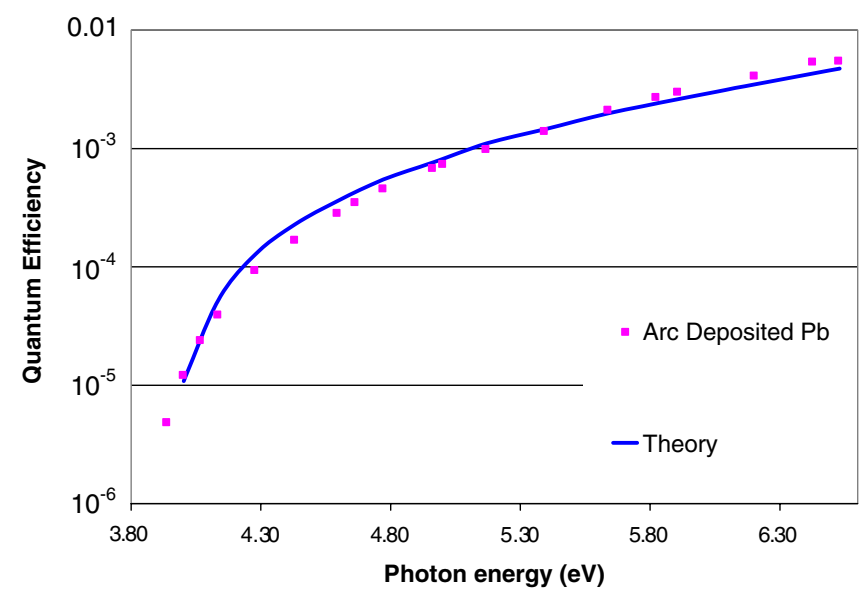

FIG. 10. (Color) Comparison between predicted and measured QE for arc-deposited lead.

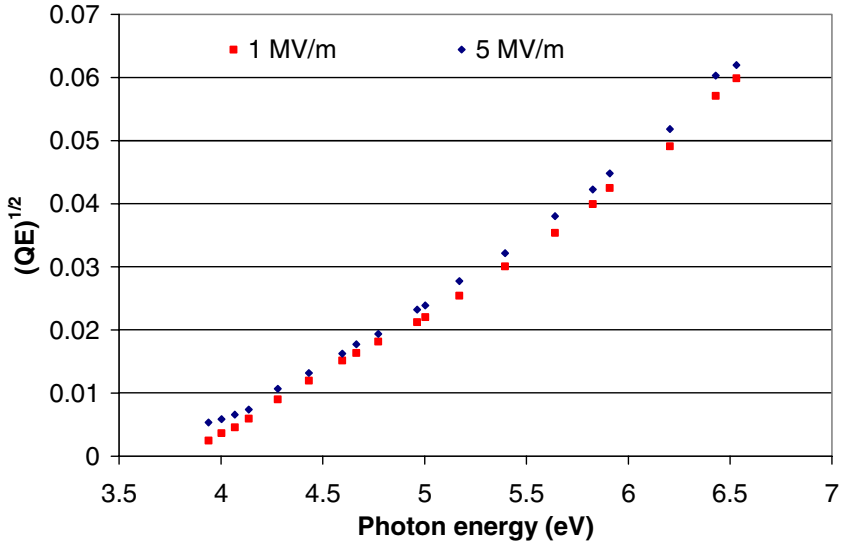

FIG. 11. (Color) $(\mathrm{QE})^{1 / 2}$ vs photon energy for sputtered lead.

included in the emission model, such as impurity of the lead, surface roughness, or surface contamination, must be involved in lowering the $\mathrm{QE}$ for these cathodes.

Figure 11 shows the data for sputtered lead plotted as $(\mathrm{QE})^{1 / 2}$ vs photon energy. This plot should be approximately a straight line, with a horizontal intercept equal to the material work function. Data for both an electrode bias of $1 \mathrm{MV} / \mathrm{m}(1 \mathrm{kV}$ across $1 \mathrm{~mm})$ and $5 \mathrm{MV} / \mathrm{m}$ are shown. The Schottky effect (the reduction of the material work function due to the applied field) is evidenced by the lower work function for the $5 \mathrm{MV} / \mathrm{m}$ data. The work functions have been calculated using the method of Fowler [16,24]; more detail on this method is below. No attempt was made

TABLE I. Work function $(\Phi)$ and $\mathrm{QE}$ of cathodes, including cleaning energy density (CED) of $248 \mathrm{~nm}$ light used, and bias field applied between the cathode and the anode. A CED of 0 represents the value prior to laser cleaning.

\begin{tabular}{llcccc}
\hline \hline & & & \multicolumn{3}{c}{ QE $\left(\times 10^{-4}\right)$} \\
Cathode & $\mathrm{CED}$ & Field & \multicolumn{2}{c}{$213 \mathrm{~nm}$} & $193 \mathrm{~nm}$ \\
\hline Solid & 0 & 1 & 4.52 & 0.49 & 2.6 \\
& 0.18 & 1 & 4.19 & 5.5 & 13.1 \\
& 0.4 & 1 & 3.93 & 7.8 & 15.3 \\
& 0.86 & 1 & 4.02 & 5.6 & 12.8 \\
\hline Evaporated & 0 & 1 & 4.22 & 0.65 & 2.9 \\
& 0 & 5 & 4.17 & 0.70 & 3.1 \\
& 0.21 & 1 & 3.97 & 15.6 & 28.4 \\
& 0.21 & 5 & 3.84 & 14.2 & 27.9 \\
& 0.37 & 1 & 3.92 & 13.3 & 25.4 \\
& 0.37 & 5 & 3.86 & 15.3 & 30.4 \\
\hline Arc & 0.21 & 1 & 3.88 & 27.2 & 54.1 \\
\hline Sputtered & 0 & 1 & 4.21 & 0.10 & 0.22 \\
& 0.23 & 1 & 3.83 & 16.0 & 32.6 \\
& 0.23 & 5 & 3.71 & 17.9 & 36.4 \\
\hline Electroplated & 0.22 & 1 & 4.20 & 6.8 & 16.0 \\
& 0.37 & 1 & 4.11 & 7.1 & 16.3 \\
\hline \hline
\end{tabular}


to account for the $2 \mathrm{~nm}$ bandwidth of the lamp light. This introduces an uncertainty of $\pm 0.04 \mathrm{eV}$ on the values for the work function. Table I shows the best fit work function for each cathode, cleaning energy, and bias field, along with the QE at 213 and $193 \mathrm{~nm}$. For some cathodes, the QE prior to cleaning was too low to be reliably measured with the lamp source; in these cases, only the QE after cleaning is shown.

\section{A. Photoemission at cryogenic temperatures}

A superconducting lead cathode will be used at cryogenic temperatures in a real injector. Tests with an allniobium injector noted a $\mathrm{QE}$ at cryogenic temperatures that was inferior to the room-temperature QE [8]. For this reason, it was instructive to investigate the $\mathrm{QE}$ of lead cathodes at cryogenic temperatures. This was accomplished by mounting the cathode on a vacuum cold finger attached to a $\mathrm{LN}_{2}$ Dewar. The cathode was electrically isolated from the thermal mass of the cold finger by a ceramic standoff, but in adequate thermal contact. A thermocouple on the thermal mass was used to monitor the cathode temperature. The cold-finger system has a $5 \mathrm{~mm}$ separation between the cathode and anode. A bias of $5 \mathrm{kV}$ was used, yielding a field of $1 \mathrm{MV} / \mathrm{m}$. The arrangement for the experiment was otherwise identical to that described above.

Both electroplated and arc-deposited samples were studied in this apparatus. The QE of each cathode was determined at room temperature in good vacuum ( 8 nTorr) before and after laser cleaning. The cathode was then cooled to $-169^{\circ} \mathrm{C}$ over the course of half an hour. Once the temperature stabilized, the cathode QE was measured again. The cathode was then laser cleaned while still cold. The QE was measured immediately after cleaning, and every half-hour afterwards. For the case of the good vacuum, the cold QE did not vary much from the room-temperature value. However, when the above protocol was repeated for "poor" vacuum conditions (1.3 $\mu$ Torr), the QE was found to degrade significantly when the cathode was cooled. These vacuum conditions were achieved by backfilling the cavity with high-purity $\mathrm{N}_{2}$, then pumping with only the turbo pump, leaving the ion pump valve closed. The room-temperature $\mathrm{QE}$ could be restored by laser cleaning, but the QE continued to degrade on the scale of hours after the cleaning. Table II shows the vacuum level, QE, and calculated work function for the arc-deposited cathode for each of the steps. In each case the QE was measured over a wide range of wavelengths; however, only the QE at 213 and $193 \mathrm{~nm}$ are shown in the Table. Note that, for the poor vacuum case, the act of cooling the cathode reduces the vacuum pressure in the cell considerably, suggesting that the cold finger is acting as a cryopump. This effect is likely the cause of the degradation of the QE in the cold, poor vacuum case-contaminants are being trapped onto the cathode. It should be noted that this is a second arc-deposited cathode, not the cathode shown in Figs. 6(a) and 7(a). The niobium substrate used for arc deposition in this case was not mechanically polished, and the lead coverage was not perfect. This cathode has a slightly higher work function, and a slightly lower $\mathrm{QE}$ than the previous cathode. The results for the electroplated cathode cryogenic test were similar to those for the arc-deposited cathode, and are not shown.

\section{B. Fowler's method}

The work functions in Tables I and II were calculated using Fowler's method [16,24]. Fowler relates the photocurrent ( $\mathrm{QE}$ in our case) to the work function $\Phi$, temperature of the electron distribution $T$, and the energy of the incident photon $(h \nu)$ using the following expression:

$$
\ln \left(\frac{\mathrm{QE}}{T^{2}}\right)=B+\ln \left[f\left(\frac{h \nu-\phi}{k_{B} T}\right)\right] .
$$

Here $B$ is a constant independent of $\nu$ and $T$. When the argument of the function $f$ is significantly larger than 1 (as it is in our case), $f$ can be approximated by the following expression:

$$
f(x)=\frac{\pi^{2}}{6}+\frac{x^{2}}{2} \quad \text { for } x \gg 1 .
$$

Figure 12 shows a plot of $\ln \left(\mathrm{QE} / T^{2}\right)$ vs $h \nu / k_{B} T$ for the data shown in Fig. 11 (sputtered lead at room temperature). The fitted lines are $B+\ln \{f[(h \nu-\Phi) / k T]\}$, with $B$ and $\Phi$

TABLE II. QE and work function for arc-deposited sample at cryogenic temperature, for both good and poor vacuum conditions.

\begin{tabular}{lrrrrr}
\hline \hline & Temperature & Vacuum & \multicolumn{3}{c}{ QE $\left(\times 10^{-4}\right)$} \\
& \multicolumn{1}{c}{$\mathrm{C}$} & nTorr & $\Phi(\mathrm{eV})$ & $213 \mathrm{~nm}$ & $193 \mathrm{~nm}$ \\
\hline Initial & 20 & 8 & 4.57 & 0.6 & 2.4 \\
Cleaned $0.23 \mathrm{~mJ} / \mathrm{mm}^{2}$ & 20 & 8 & 4.00 & 10.1 & 26.0 \\
Cold & -169 & 6 & 4.00 & 10.1 & 26.0 \\
Poor vac & 20 & 1300 & 4.04 & 8.6 & 23.5 \\
Cold & -169 & 270 & 4.45 & 3.0 & 8.5 \\
Cleaned $0.22 \mathrm{~mJ} / \mathrm{mm}^{2}$ & -169 & 270 & 4.06 & 10.0 & 24.4 \\
2 hrs later & -169 & 270 & 4.26 & 4.6 & 11.1 \\
\hline \hline
\end{tabular}




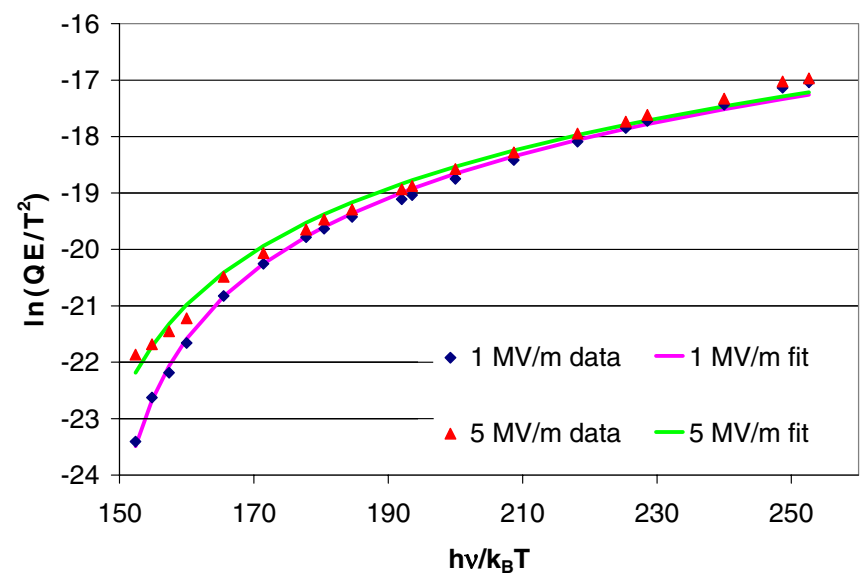

FIG. 12. (Color) Plot of $\ln \left(\mathrm{QE} / T^{2}\right)$ vs $h \nu / k_{B} T$ for sputtered lead cathode after laser cleaning, for both 1 and $5 \mathrm{MV} / \mathrm{m}$ field.

as free parameters. Although $B$ was allowed to vary independently for both data sets, the best fit value for $B$ was unchanged by the change in field, while the value for $\Phi$ was reduced by increasing the field, as expected from the Schottky effect.

\section{CONCLUSION}

Lead appears to be an attractive option for moderate average current sources. The best measured QE (arcdeposited) would require $2.1 \mathrm{~W}$ at $213 \mathrm{~nm}$ to generate $1 \mathrm{~mA}$. The optimal choice for a lead coating method seems to be arc deposition, as it provides a smooth surface [Figs. 6(a) and 7(a)] and good QE. Electroplating is also an option, although the electroplated samples we tested had a work function significantly higher than the other lead samples, and correspondingly lower QE. The surface finishes of the evaporated and sputtered samples are likely too rough to use in an injector, at least at high field.

The three-step model of photoemission seems to well predict the QE of lead, especially given that no free parameters are used in the model. The work functions of the lead samples agree with the expected value, with the exception of the electroplated sample. Modest laser cleaning energy densities $\left(\sim 0.2 \mathrm{~mJ} / \mathrm{mm}^{2}\right)$ are sufficient to achieve the maximum QE without damage to the coating. Cryogenic QE is consistent with room-temperature QE; however, poor vacuum conditions ( $\sim 1 \mu$ Torr) lead to rapid loss of $\mathrm{QE}$ in cryogenic conditions.

\section{ACKNOWLEDGMENTS}

The authors would like to thank R. Lefferts and A. Lipski of Stonybrook University for preparing the electroplated and evaporated lead samples, and J. Langner and P. Strzyżewski of Andrzej Soltan Institute for Nuclear Studies for preparing the sputtered and arc-deposited samples. The authors are indebted to J. Warren for the scanning electron microscope pictures, and to K. Jensen for invaluable discussions on the process of electron scattering. The authors acknowledge J. Walsh for his expert technical assistance and V. Radeka for his support. This manuscript has been authored by Brookhaven Science Associates, LLC under Contract No. DE-AC0298CH10886 with the U.S. Department of Energy.

[1] J. Sekutowicz et al., Phys. Rev. ST Accel. Beams 8, 010701 (2005).

[2] I. Ben-Zvi et al., Proceedings of 2005 Particle Accelerator Conference, Knoxville, Tennessee (IEEE, Piscataway, NJ, 2005), pp. 1150 and 2741.

[3] V. N. Litvinenko et al., Proceedings of 2005 Particle Accelerator Conference, Knoxville, Tennessee (Ref. [2], p. 2767).

[4] Several existing and planned Linac-based photon sources require high-average current photoinjectors, such as the operating FEL at Jefferson Laboratory (http://www.jlab. org/FEL), and the proposed light sources at Cornell (http:// erl.chess.cornell.edu) and Daresbury (http://www.4gls. ac.uk/.

[5] A. Michalke, H. Piel, C. Sinclair, and P. Michelato, Proceedings of 1992 European Particle Accelerator Conference, Berlin, Germany (1992), p. 1014.

[6] D. Janssen et al., Nucl. Instrum. Methods Phys. Res., Sect. A 507, 314 (2003).

[7] X. Chang et al., Proceedings of 2005 Particle Accelerator Conference, Knoxville, Tennessee (Ref. [2], p. 2251).

[8] T. Rao et al., Proceedings of 2005 Particle Accelerator Conference, Knoxville, Tennessee (Ref. [2], p. 2556).

[9] J. Smedley, T. Rao, and Q. Zhao, J. Appl. Phys. 98, 043111 (2005).

[10] J. Smedley, T. Rao, J. Warren, J. Sekutowicz, R. Lefferts, and A. Lipski, Proceedings of 2004 European Particle Accelerator Conference, Lucerne, Switzerland (2004), p. 1126.

[11] J. Sekutowicz et al., DESY TESLA-FEL Report 2005-09, Hamburg, Germany.

[12] J. Sekutowicz et al., Status of $\mathrm{Nb}$-Pb Superconducting RFGun Cavities, Proceedings of 2007 Particle Accelerator Conference, Albuquerque, New Mexico (IEEE, Piscataway, NJ, 2007), p. 962.

[13] W. F. Krolikowski and W.E. Spicer, Phys. Rev. 185, 882 (1969).

[14] J. Smedley et al., Superconducting Photocathodes; Proceedings of Workshop on the Physics and Applications of High Brightness Electron Beams, Erice, Sicily, 2005. This paper was intended for a special section of PRST-AB devoted to this workshop.

[15] D.H. Dowell, Models for Calculating the $Q E$ and Thermal Emittance; Proceedings of the 1st Workshop on High Quantum Efficiency Photocathodes for $\mathrm{rf}$ Guns, Milan, Italy (2006), http://wwwlasa.mi.infn.it/ WSPhotocathodes/uploaded-files/sem-thermal-emittance-i/ DowellHighQEtalkFinal.pdf.

[16] D. H. Dowell, F. K. King, R. E. Kirby, J. F. Schmerge, and J. M. Smedley, Phys. Rev. ST Accel. Beams 9, 063502 (2006). 
[17] K. L. Jensen, D. W. Feldman, E. J. Montgomery, P. G. O'Shea, and N. A. Moody (to be published).

[18] Naval Research Laboratory Structure Database, http://cstwww.nrl.navy.mil/ElectronicStructureDatabase.

[19] C. Norris and L. Wallden, J. Phys. F 2, 180 (1972).

[20] H. G. Liljenvall, A.G. Mathewson, and H.P. Myers, Philos. Mag. 22, 243 (1970).

[21] A. V. Lugovskoy and I. Bray, Phys. Rev. B 60, 3279
(1999).

[22] J.W. Noe, Proceeding of the Heavy Ion Accelerator Technology, 8th International Conference (American Institute of Physics, Melville, New York, 1999), p. 192.

[23] LeaRonal Solderon from Shipely Company, LLC, Marborough, MA 01752.

[24] R. H. Fowler, Phys. Rev. 38, 45 (1931). 